\title{
Recent developments in QCD thermodynamics and collective excitations from hard-thermal-loop effective theory
}

\author{
Nan $\mathrm{Su}^{1, \mathrm{a}}$ \\ ${ }^{1}$ Frankfurt Institute for Advanced Studies, 60438 Frankfurt am Main, Germany
}

\begin{abstract}
I summarize recent developments in the hard-thermal-loop approach to QCD. I first discuss a finite-temperature and -density calculation of QCD thermodynamics at NNLO from the hard-thermal-loop perturbation theory. I then discuss a generalization of the hard-thermal-loop framework to the magnetic scale $g^{2} T$, from which a novel nonAbelian massless mode is uncovered.
\end{abstract}

\section{Introduction}

In this proceedings, I overview recent developments in QCD thermodynamics and collective excitations from the hard-thermal-loop (HTL) effective theory. This is an active research area driven especially by the pending challenges in the study of the quark-gluon plasma (QGP) from the ultrarelativistic heavy-ion collisions currently carried out at RHIC (BNL, USA), LHC (CERN, Switzerland), and forthcoming ones at FAIR (GSI, Germany). The QCD running coupling expected in the experimental energies is on the order of unity $g \sim O(1)$, which is some intermediate value neither huge nor tiny. Besides the intrinsic scale temperature $T$, collective behaviors of the QGP generate two thermal scales, namely the electric scale $g T$ (perturbative) and the magnetic scale $g^{2} T$ (non-perturbative), which have different physical origins. These subtleties make it extremely challenging to tackle the QGP in and out of equilibrium in the phenomenologically relevant regime where $g \sim O(1)$ using the continuum QCD method of resummed thermal perturbation theory. For this concern, I summarize in the following recent developments from the HTL effective theory focusing on thermodynamics from the electric scale $g T$ and collective excitations from the magnetic scale $g^{2} T$ (see Ref. [1] for a review).

\section{Electric scale $g T:$ NNLO thermodynamics from hard-thermal-loop perturbation theory}

The hard-thermal-loop effective theory is the resummation addressing the physics of the electric scale $g T[2,3]$. Hard-thermal-loop perturbation theory (HTLpt) is a reorganization of thermal QCD that improves the convergence of the perturbation expansion with the help of the HTL effective action [4]. The Minkowskian HTLpt Lagrangian density reads

$$
\mathcal{L}=\left.\left(\mathcal{L}_{\mathrm{QCD}}+\mathcal{L}_{\mathrm{HTL}}\right)\right|_{g \rightarrow \sqrt{\delta} g}+\Delta \mathcal{L}_{\mathrm{HTL}}
$$

\footnotetext{
a e-mail: nansu@ fias.uni-frankfurt.de
} 
Here $\mathcal{L}_{\mathrm{QCD}}$ is the QCD Lagrangian density that reads

$$
\mathcal{L}_{\mathrm{QCD}}=-\frac{1}{2} \operatorname{Tr}\left[G_{\mu \nu} G^{\mu \nu}\right]+i \bar{\psi} \gamma^{\mu} D_{\mu} \psi+\mathcal{L}_{\mathrm{gh}}+\mathcal{L}_{\mathrm{gf}}+\Delta \mathcal{L}_{\mathrm{QCD}},
$$

where $G^{\mu \nu}=\partial^{\mu} A^{v}-\partial^{v} A^{\mu}-i g\left[A^{\mu}, A^{v}\right]$ is the gluon field strength tensor, $D^{\mu}=\partial^{\mu}-i g A^{\mu}$ is the covariant derivative, and $\psi$ is the quark field. The term $\Delta \mathcal{L}_{\mathrm{QCD}}$ contains all the standard QCD counterterms. The ghost term $\mathcal{L}_{\text {gh }}$ depends on the form of the gauge-fixing term $\mathcal{L}_{\text {gf }}$.

The HTL improvement term $\mathcal{L}_{\text {HTL }}$ reads

$$
\mathcal{L}_{\mathrm{HTL}}=(1-\delta) i m_{q}^{2} \bar{\psi} \gamma^{\mu}\left\langle\frac{y_{\mu}}{y \cdot D}\right\rangle_{\hat{\mathbf{y}}} \psi-\frac{1}{2}(1-\delta) m_{D}^{2} \operatorname{Tr}\left(G_{\mu \alpha}\left\langle\frac{y^{\alpha} y_{\beta}}{(y \cdot D)^{2}}\right\rangle_{\hat{\mathbf{y}}} G^{\mu \beta}\right),
$$

where $y^{\mu}=(1, \hat{\mathbf{y}})$ is a light-like four-vector and the angular bracket indicates an average over the direction of $\hat{\mathbf{y}}$. The two parameters $m_{D}$ and $m_{q}$ can be identified with the Debye screening mass and the quark thermal mass, respectively. HTLpt is defined by treating $\delta$ as a formal expansion parameter. By coupling the HTL improvement term (3) to the QCD Lagrangian (2), HTLpt systematically shifts the perturbative expansion from being around an ideal gas of massless particles to being around an ideal gas of massive quasiparticles which are more appropriate physical degrees of freedom at high temperature and/or density.

Physical observables are calculated in HTLpt by expanding in powers of $\delta$, truncating at some specified order, and then setting $\delta=1$. This defines a reorganization of the perturbative series in which the effects of the $m_{D}^{2}$ and $m_{q}^{2}$ terms in (3) are included to leading order but then systematically subtracted out at higher orders in perturbation theory by the $\delta m_{D}^{2}$ and $\delta m_{q}^{2}$ terms in (3). The HTLpt Lagrangian (1) reduces to the QCD Lagrangian (2) when we set $\delta=1$. If the expansion in $\delta$ could be calculated to all orders, the final result would not depend on $m_{D}$ and $m_{q}$ when we set $\delta=1$. However, any truncation of the expansion in $\delta$ produces results that depend on $m_{D}$ and $m_{q}$. As a consequence, a prescription is required to determine $m_{D}$ and $m_{q}$ as a function of $T, \mu$ and $\alpha_{s}$. Note that HTLpt is gauge invariant order-by-order in the $\delta$ expansion.

The HTLpt expansion generates additional ultraviolet divergences. In QCD perturbation theory, renormalizability constrains the UV divergences to have a form that can be cancelled by the counterterm Lagrangian $\Delta \mathcal{L}_{\mathrm{QCD}}$. There is yet no proof for the renormalizability of the HTL perturbation expansion, it has been shown in Refs. [5-13] that it is possible to renormalize the HTLpt thermodynamic potential through NNLO with a counterterm Lagrangian $\Delta \mathcal{L}_{\text {HTL }}$ containing only a vacuum counterterm, a Debye mass counterterm, a fermion mass counterterm, and a coupling constant counterterm, as listed below

$$
\begin{aligned}
\Delta \mathcal{E}_{0} & =\frac{d_{A}}{128 \pi^{2} \epsilon}(1-\delta)^{2} m_{D}^{4}, \\
\Delta m_{D}^{2} & =\frac{11 c_{A}-4 s_{F}}{12 \pi \epsilon} \alpha_{s} \delta(1-\delta) m_{D}^{2}, \\
\Delta m_{q}^{2} & =\frac{3}{8 \pi \epsilon} \frac{d_{A}}{c_{A}} \alpha_{S} \delta(1-\delta) m_{q}^{2}, \\
\delta \Delta \alpha_{s} & =-\frac{11 c_{A}-4 s_{F}}{12 \pi \epsilon} \alpha_{s}^{2} \delta^{2},
\end{aligned}
$$

where $c_{A}=N_{c}, d_{A}=N_{c}^{2}-1, s_{F}=N_{f} / 2, d_{F}=N_{c} N_{f}$, and $s_{2 F}=C_{F} s_{f}$ with $C_{F}=\left(N_{c}^{2}-1\right) / 2 N_{c}$. Note that the coupling constant counterterm (7) is consistent with one-loop running of $\alpha_{s}$.

The calculation of the thermodynamic potential in HTLpt involves the evaluation of vacuum diagrams. Defining $\boldsymbol{\aleph}(z) \equiv \Psi(z)+\Psi\left(z^{*}\right)$ with $z=1 / 2-i \hat{\mu}$ and $\Psi$ being the digamma function, 
$\Omega_{0} \equiv-d_{A} \pi^{2} T^{4} / 45$, and $\hat{x} \equiv x / 2 \pi T$ for dimensionless variables, the NNLO thermodynamic potential for QCD at finite temperature $T$ and density/chemical potential $\mu$ (with $\mu_{f}$ being the chemical potential for quarks with flavor $f$ ) reads [13]

$$
\begin{aligned}
& \frac{\Omega_{\mathrm{NNLO}}}{\Omega_{0}}=\frac{7}{4} \frac{d_{F}}{d_{A}} \frac{1}{N_{f}} \sum_{f}\left(1+\frac{120}{7} \hat{\mu}_{f}^{2}+\frac{240}{7} \hat{\mu}_{f}^{4}\right)-\frac{s_{F} \alpha_{s}}{\pi} \frac{1}{N_{f}} \sum_{f}\left[\frac{5}{8}\left(1+12 \hat{\mu}_{f}^{2}\right)\left(5+12 \hat{\mu}_{f}^{2}\right)\right. \\
& \left.-\frac{15}{2}\left(1+12 \hat{\mu}_{f}^{2}\right) \hat{m}_{D}-\frac{15}{2}\left(2 \ln \frac{\hat{\Lambda}}{2}-1-\aleph\left(z_{f}\right)\right) \hat{m}_{D}^{3}+90 \hat{m}_{q}^{2} \hat{m}_{D}\right] \\
& +\frac{s_{2 F}}{N_{f}}\left(\frac{\alpha_{s}}{\pi}\right)^{2} \sum_{f}\left[\frac { 1 5 } { 6 4 } \left\{35-32\left(1-12 \hat{\mu}_{f}^{2}\right) \frac{\zeta^{\prime}(-1)}{\zeta(-1)}+472 \hat{\mu}_{f}^{2}+1328 \hat{\mu}_{f}^{4}\right.\right. \\
& \left.+64\left(-36 i \hat{\mu}_{f} \boldsymbol{\aleph}\left(2, z_{f}\right)+6\left(1+8 \hat{\mu}_{f}^{2}\right) \boldsymbol{\aleph}\left(1, z_{f}\right)+3 i \hat{\mu}_{f}\left(1+4 \hat{\mu}_{f}^{2}\right) \boldsymbol{\aleph}\left(0, z_{f}\right)\right)\right\} \\
& \left.-\frac{45}{2} \hat{m}_{D}\left(1+12 \hat{\mu}_{f}^{2}\right)\right] \\
& +\left(\frac{s_{F} \alpha_{s}}{\pi}\right)^{2} \frac{1}{N_{f}} \sum_{f} \frac{5}{16}\left[96\left(1+12 \hat{\mu}_{f}^{2}\right) \frac{\hat{m}_{q}^{2}}{\hat{m}_{D}}+\frac{4}{3}\left(1+12 \hat{\mu}_{f}^{2}\right)\left(5+12 \hat{\mu}_{f}^{2}\right) \ln \frac{\hat{\Lambda}}{2}\right. \\
& +\frac{1}{3}+4 \gamma_{E}+8\left(7+12 \gamma_{E}\right) \hat{\mu}_{f}^{2}+112 \mu_{f}^{4}-\frac{64}{15} \frac{\zeta^{\prime}(-3)}{\zeta(-3)}-\frac{32}{3}\left(1+12 \hat{\mu}_{f}^{2}\right) \frac{\zeta^{\prime}(-1)}{\zeta(-1)} \\
& \left.-96\left\{8 \boldsymbol{\kappa}\left(3, z_{f}\right)+12 i \hat{\mu}_{f} \boldsymbol{\kappa}\left(2, z_{f}\right)-2\left(1+2 \hat{\mu}_{f}^{2}\right) \boldsymbol{\kappa}\left(1, z_{f}\right)-i \hat{\mu}_{f} \boldsymbol{\aleph}\left(0, z_{f}\right)\right\}\right] \\
& +\left(\frac{s_{F} \alpha_{s}}{\pi}\right)^{2} \frac{1}{N_{f}^{2}} \sum_{f, g}\left[\frac{5}{4 \hat{m}_{D}}\left(1+12 \hat{\mu}_{f}^{2}\right)\left(1+12 \hat{\mu}_{g}^{2}\right)+90\left\{2\left(1+\gamma_{E}\right) \hat{\mu}_{f}^{2} \hat{\mu}_{g}^{2}\right.\right. \\
& -\left\{\boldsymbol{\aleph}\left(3, z_{f}+z_{g}\right)+\boldsymbol{\aleph}\left(3, z_{f}+z_{g}^{*}\right)+4 i \hat{\mu}_{f}\left[\boldsymbol{\aleph}\left(2, z_{f}+z_{g}\right)+\boldsymbol{\aleph}\left(2, z_{f}+z_{g}^{*}\right)\right]-4 \hat{\mu}_{g}^{2} \boldsymbol{\aleph}\left(1, z_{f}\right)\right. \\
& \left.\left.-\left(\hat{\mu}_{f}+\hat{\mu}_{g}\right)^{2} \boldsymbol{\aleph}\left(1, z_{f}+z_{g}\right)-\left(\hat{\mu}_{f}-\hat{\mu}_{g}\right)^{2} \boldsymbol{\aleph}\left(1, z_{f}+z_{g}^{*}\right)-4 i \hat{\mu}_{f} \hat{\mu}_{g}^{2} \boldsymbol{\aleph}\left(0, z_{f}\right)\right\}\right\} \\
& \left.-\frac{15}{2}\left(1+12 \hat{\mu}_{f}^{2}\right)\left(2 \ln \frac{\hat{\Lambda}}{2}-1-\aleph\left(z_{g}\right)\right) \hat{m}_{D}\right] \\
& +\left(\frac{c_{A} \alpha_{s}}{3 \pi}\right)\left(\frac{s_{F} \alpha_{s}}{\pi N_{f}}\right) \sum_{f}\left[\frac{15}{2 \hat{m}_{D}}\left(1+12 \hat{\mu}_{f}^{2}\right)-\frac{235}{16}\left\{\left(1+\frac{792}{47} \hat{\mu}_{f}^{2}+\frac{1584}{47} \hat{\mu}_{f}^{4}\right) \ln \frac{\hat{\Lambda}}{2}\right.\right. \\
& -\frac{144}{47}\left(1+12 \hat{\mu}_{f}^{2}\right) \ln \hat{m}_{D}+\frac{319}{940}\left(1+\frac{2040}{319} \hat{\mu}_{f}^{2}+\frac{38640}{319} \hat{\mu}_{f}^{4}\right)-\frac{24 \gamma_{E}}{47}\left(1+12 \hat{\mu}_{f}^{2}\right) \\
& -\frac{44}{47}\left(1+\frac{156}{11} \hat{\mu}_{f}^{2}\right) \frac{\zeta^{\prime}(-1)}{\zeta(-1)}-\frac{268}{235} \frac{\zeta^{\prime}(-3)}{\zeta(-3)}-\frac{72}{47}\left[4 i \hat{\mu}_{f} \boldsymbol{\aleph}\left(0, z_{f}\right)+\left(5-92 \hat{\mu}_{f}^{2}\right) \boldsymbol{\kappa}\left(1, z_{f}\right)\right. \\
& \left.\left.+144 i \hat{\mu}_{f} \aleph\left(2, z_{f}\right)+52 \boldsymbol{\aleph}\left(3, z_{f}\right)\right]\right\}+90 \frac{\hat{m}_{q}^{2}}{\hat{m}_{D}}+\frac{315}{4}\left\{\left(1+\frac{132}{7} \hat{\mu}_{f}^{2}\right) \ln \frac{\hat{\Lambda}}{2}\right. \\
& \left.\left.+\frac{11}{7}\left(1+12 \hat{\mu}_{f}^{2}\right) \gamma_{E}+\frac{9}{14}\left(1+\frac{132}{9} \hat{\mu}_{f}^{2}\right)+\frac{2}{7} \boldsymbol{\aleph}\left(z_{f}\right)\right\} \hat{m}_{D}\right]+\frac{\Omega_{\mathrm{NNLO}}^{\mathrm{YM}}}{\Omega_{0}},
\end{aligned}
$$

where $\Omega_{\mathrm{NNLO}}^{\mathrm{YM}}$ is the pure-glue contribution to the thermodynamic potential that reads [6] 


$$
\begin{aligned}
\frac{\Omega_{\mathrm{NNLO}}^{\mathrm{YM}}}{\Omega_{0}}= & 1-\frac{15}{4} \hat{m}_{D}^{3}+\frac{N_{c} \alpha_{s}}{3 \pi}\left[-\frac{15}{4}+\frac{45}{2} \hat{m}_{D}-\frac{135}{2} \hat{m}_{D}^{2}-\frac{495}{4}\left(\ln \frac{\hat{\Lambda}}{2}+\frac{5}{22}+\gamma_{E}\right) \hat{m}_{D}^{3}\right] \\
& +\left(\frac{N_{c} \alpha_{s}}{3 \pi}\right)^{2}\left[\frac{45}{4 \hat{m}_{D}}-\frac{165}{8}\left(\ln \frac{\hat{\Lambda}}{2}-\frac{72}{11} \ln \hat{m}_{D}-\frac{84}{55}-\frac{6}{11} \gamma_{E}-\frac{74}{11} \frac{\zeta^{\prime}(-1)}{\zeta(-1)}\right.\right. \\
& \left.\left.+\frac{19}{11} \frac{\zeta^{\prime}(-3)}{\zeta(-3)}\right)+\frac{1485}{4}\left(\ln \frac{\hat{\Lambda}}{2}-\frac{79}{44}+\gamma_{E}+\log 2-\frac{\pi^{2}}{11}\right) \hat{m}_{D}\right] .
\end{aligned}
$$

As discussed in Ref. [9], the two-loop perturbative electric mass for gluons introduced by Braaten and Nieto in Refs. $[14,15]$ originally at finite $T$ and vanishing $\mu$ is the most suitable one for NNLO HTLpt calculations. The finite $T$ and $\mu$ generalization was obtained in Ref. [16] that reads

$$
\begin{aligned}
\hat{m}_{D}^{2}= & \frac{\alpha_{s}}{3 \pi}\left\{c_{A}+\frac{c_{A}^{2} \alpha_{s}}{12 \pi}\left(5+22 \gamma_{E}+22 \ln \frac{\hat{\Lambda}_{g}}{2}\right)+\frac{1}{N_{f}} \sum_{f}\left[s_{F}\left(1+12 \hat{\mu}_{f}^{2}\right)\right.\right. \\
& +\frac{c_{A} s_{F} \alpha_{s}}{12 \pi}\left(\left(9+132 \hat{\mu}_{f}^{2}\right)+22\left(1+12 \hat{\mu}_{f}^{2}\right) \gamma_{E}+2\left(7+132 \hat{\mu}_{f}^{2}\right) \ln \frac{\hat{\Lambda}}{2}+4 \boldsymbol{\aleph}\left(z_{f}\right)\right) \\
& \left.\left.+\frac{s_{F}^{2} \alpha_{s}}{3 \pi}\left(1+12 \hat{\mu}_{f}^{2}\right)\left(1-2 \ln \frac{\hat{\Lambda}}{2}+\boldsymbol{\kappa}\left(z_{f}\right)\right)-\frac{3}{2} \frac{s_{2 F} \alpha_{s}}{\pi}\left(1+12 \hat{\mu}_{f}^{2}\right)\right]\right\} .
\end{aligned}
$$

The effect of the in-medium quark mass parameter $m_{q}$ in thermodynamic functions is small and it is thus set to 0 which is the three-loop variational solution for $m_{q}$ following Ref. [9].

By taking derivatives of the thermodynamic potential (8) with respect to $T$ or $\mu$ (and then setting $\mu=0$ ), one can generate all the thermodynamic functions as well as quark and baryon number susceptibilities. Sec. 6 in Ref. [13] presents detailed comparisons of various NNLO thermodynamic functions - including the pressure, energy density, entropy density, trace anomaly, and speed of sound - with state-of-the-art lattice data, and very good agreements are obtained in the phenomenologically relevant regime. Sec. 7 in Ref. [13] presents detailed comparisons of various NNLO quark and baryon number susceptibilities that also show encouraging signals in the phenomenologically relevant regime. Note that there has been recent progress for the same course of study from dimensional reduction $[17,18]$. The NNLO thermodynamic calculations have set the stage for real-time applications of HTLpt.

\section{Magnetic scale $g^{2} T$ : collective excitations from hard-thermal-loop approach to Gribov-Zwanziger quantization}

Conventional (resummed) thermal perturbation theory breaks down at the magnetic scale $g^{2} T$ due to the so-called Linde problem $[19,20]$. The non-perturbativeness of the magnetic scale is closely related to the confining nature of the dimensionally reduced Yang-Mills theory at high $T$. This suggests the need of incorporating a confinement mechanism in perturbative expansions even when dealing with the deconfined QGP phase. A formalism to tackle this issue is the Gribov-Zwanziger (GZ) quantization from the study of color confinement [21, 22]. It regulates the IR behavior of QCD by fixing the residual gauge transformations, i.e., Gribov copies, that remain after applying the FaddeevPopov procedure. The GZ action is renormalizable, therefore it provides a systematic framework for perturbative calculations (i.e., $g \ll 1$ ) incorporating confinement effects. The gluon propagator in Landau gauge reads

$$
D^{\mu \nu}(P)=\left[\delta^{\mu \nu}-\frac{P^{\mu} P^{v}}{P^{2}}\right] \frac{P^{2}}{P^{4}+\gamma_{G}^{4}},
$$


where $\gamma_{G}$ is the Gribov parameter. $\gamma_{G}$ is solved self-consistently from a gap equation defined to infinite loop orders. Eq. (11) is IR suppressed, manifesting confinement effects, and it is a significant improvement over the one from the Faddeev-Popov quantization. The gap equation at one-loop order can be solved analytically at asymptotically high $T$ and gives [23]

$$
\gamma_{G}=\frac{D-1}{D} \frac{N_{c}}{4 \sqrt{2} \pi} g^{2} T,
$$

where $D$ is the space-time dimensions. Eq. (12) provides a fundamental IR cutoff at the magnetic scale for the finite- $T$ GZ action.

Self-energies of quarks and gluons are important measures for the collective behavior of the QGP, since thermal masses, dispersion relations, and spectral functions of collective excitations are derived from them. The Euclidean one-loop quark self-energy reads

$$
\Sigma(P)=(i g)^{2} C_{F} \oint_{\{K\}} \gamma^{\mu} S(K) \gamma^{v} D^{\mu \nu}(P-K),
$$

where $S(P)$ is the quark propagator and $D^{\mu \nu}(P)$ is the gluon propagator taken from Eq. (11). It is worth noting that there have been similar studies for the quark self-energy with non-perturbative gluons at finite density [24, 25] and in strong magnetic fields [26].

At $g \ll 1$ (i.e., high $T$ ), we may apply the hard-thermal-loop systematics in analyzing Eq. (13). As a result, the gauge-invariant contribution to Eq. (13) reads [27]

$$
\begin{aligned}
\Sigma(P) \simeq & -(i g)^{2} C_{F} \sum_{ \pm} \int_{0}^{\infty} \frac{\mathrm{d} k}{2 \pi^{2}} k^{2} \int \frac{\mathrm{d} \Omega}{4 \pi} \frac{\tilde{n}_{ \pm}\left(k, \gamma_{G}\right)}{4 E_{ \pm}^{0}} \\
& \times\left[\frac{i \gamma_{0}+\hat{\boldsymbol{k}} \cdot \boldsymbol{\gamma}}{i P_{0}+k-E_{ \pm}^{0}+\frac{\boldsymbol{p} \cdot \boldsymbol{k}}{E_{ \pm}^{0}}}+\frac{i \gamma_{0}-\hat{\boldsymbol{k}} \cdot \boldsymbol{\gamma}}{i P_{0}-k+E_{ \pm}^{0}-\frac{\boldsymbol{p} \cdot \boldsymbol{k}}{E_{ \pm}^{0}}}\right],
\end{aligned}
$$

where $\hat{\boldsymbol{k}}=\boldsymbol{k} / k$ with $k=|\boldsymbol{k}|, E_{ \pm}^{0}=\sqrt{k^{2} \pm i \gamma_{G}^{2}}, \tilde{n}_{ \pm}\left(k, \gamma_{G}\right) \equiv n_{B}\left(\sqrt{k^{2} \pm i \gamma_{G}^{2}}\right)+n_{F}(k)$ with $n_{B}$ and $n_{F}$ the Bose-Einstein and Fermi-Dirac distributions, and $\int \mathrm{d} \Omega=\int_{0}^{2 \pi} \mathrm{d} \phi \int_{0}^{\pi} \mathrm{d} \cos \theta$.

The quark thermal mass incorporating effects from $g^{2} T$ reads

$$
m_{q}^{2}\left(\gamma_{G}\right)=\frac{g^{2} C_{F}}{4 \pi^{2}} \sum_{ \pm} \int_{0}^{\infty} d k \frac{k^{2} \tilde{n}_{ \pm}\left(k, \gamma_{G}\right)}{E_{ \pm}^{0}}
$$

It reduces to the conventional HTL one, $m_{q}^{2}(0)=C_{F} g^{2} T^{2} / 8$, when setting $\gamma_{G}=0 . m_{q}^{2}\left(\gamma_{G}\right)$ receives negative contributions from $\gamma_{G}$, which is a manifestation of anti-screening effects generated by $g^{2} T$ (see Fig. 1 in Ref. [27] for details). This is a profound signal of the build-up of long-range correlations in the system.

The dispersion relation is obtained by analytically continuing the self-energy (14) to Minkowski space and then solving the poles in the corresponding quark propagator $i S^{-1}(P)=\not P-\Sigma(P)=0$. In contrast to the conventional HTL case, there are three poles in the propagator (see Fig. 2 in Ref. [27] for details). Firstly, the screened quasi-particle excitations are recovered,

$$
\omega=\omega_{+}\left(p ; \gamma_{G}\right), \quad \omega=\omega_{-}\left(p ; \gamma_{G}\right),
$$

which are the so-called particle $\omega_{+}$and plasmino $\omega_{-}$modes, with $\omega_{ \pm}\left(0 ; \gamma_{G}\right)=m_{q}\left(\gamma_{G}\right)$ as expected. Both $\omega_{ \pm} / m_{q}\left(\gamma_{G}\right)$ and their residues $Z_{ \pm}$are $g$-independent in the studied range. This property is identical to the conventional HTL case, which provides a non-trivial consistency check of the setup. Furthermore, there exists a novel excitation named Gribov pole as in Ref. [27],

$$
\omega=\omega_{G}\left(p ; \gamma_{G}\right)
$$


It describes massless fermionic excitations in the QGP with dispersion relation $\omega=v_{s} p$ at small momenta, with $v_{s} \approx 1 / \sqrt{3}$ (speed of sound) independent of $g$ for the studied range. The Gribov mode "grows" in the $(\omega, p)$-plane while increasing the magnetic scale, and this effectively introduces a new magnetic scaling behavior to the non-Abelian plasma. At larger momenta than the permitted ones for each coupling, we hit branch cuts and Landau damping consequently takes place. The Gribov pole goes along with a residue $Z_{G}(p)<0$ that induces positivity violation in the corresponding spectral functions in the region of space-like momenta. These novel features are direct manifestations of longrange confinement effects surviving at finite $T$ in the non-Abelian plasma. The results reflect common features of Gribov-like approaches [21, 22, 28], though the calculation was done via the GZ action. It is tempting to explore the impact of the setup to heavy-ion phenomenology [29-32].

\section{Acknowledgments}

I acknowledge Jens Andersen, Aritra Bandyopadhyay, Najmul Haque, Sylvain Mogliacci, Munshi Mustafa, Michael Strickland, Konrad Tywoniuk, and Aleksi Vuorinen for collaborations on which this proceedings is based. I am thankful to the support from the GSI Helmholtzzentrum für Schwerionenforschung and Horst Stöcker.

\section{References}

[1] N. Su, Int. J. Mod. Phys. A 30, 1530025 (2015).

[2] J. Frenkel and J. C. Taylor, Nucl. Phys. B 334, 199 (1990).

[3] E. Braaten and R. D. Pisarski, Nucl. Phys. B 337, 569 (1990).

[4] J. O. Andersen, E. Braaten and M. Strickland, Phys. Rev. Lett. 83, 2139 (1999).

[5] J. O. Andersen, M. Strickland and N. Su, Phys. Rev. D 80, 085015 (2009).

[6] J. O. Andersen, M. Strickland and N. Su, Phys. Rev. Lett. 104, 122003 (2010).

[7] J. O. Andersen, M. Strickland and N. Su, JHEP 1008, 113 (2010).

[8] J. O. Andersen, L. E. Leganger, M. Strickland and N. Su, Phys. Lett. B 696, 468 (2011).

[9] J. O. Andersen, L. E. Leganger, M. Strickland and N. Su, JHEP 1108, 053 (2011).

[10] N. Su, arXiv:1104.3450 [hep-ph].

[11] N. Su, Commun. Theor. Phys. 57, 409 (2012).

[12] N. Haque, J. O. Andersen, M. G. Mustafa, M. Strickland and N. Su, Phys. Rev. D 89, 061701 (2014).

[13] N. Haque, A. Bandyopadhyay, J. O. Andersen, M. G. Mustafa, M. Strickland and N. Su, JHEP 1405, 027 (2014).

[14] E. Braaten and A. Nieto, Phys. Rev. Lett. 76, 1417 (1996).

[15] E. Braaten and A. Nieto, Phys. Rev. D 53, 3421 (1996).

[16] A. Vuorinen, Phys. Rev. D 68, 054017 (2003).

[17] J. O. Andersen, S. Mogliacci, N. Su and A. Vuorinen, Phys. Rev. D 87, 074003 (2013).

[18] S. Mogliacci, J. O. Andersen, M. Strickland, N. Su and A. Vuorinen, JHEP 1312, 055 (2013).

[19] A. D. Linde, Phys. Lett. B 96, 289 (1980).

[20] D. J. Gross, R. D. Pisarski and L. G. Yaffe, Rev. Mod. Phys. 53, 43 (1981).

[21] V. N. Gribov, Nucl. Phys. B 139, 1 (1978).

[22] D. Zwanziger, Nucl. Phys. B 323, 513 (1989).

[23] K. Fukushima and N. Su, Phys. Rev. D 88, 076008 (2013). 
[24] T. Kojo, Y. Hidaka, L. McLerran and R. D. Pisarski, Nucl. Phys. A 843, 37 (2010).

[25] T. Kojo, Y. Hidaka, K. Fukushima, L. D. McLerran and R. D. Pisarski, Nucl. Phys. A 875, 94 (2012).

[26] T. Kojo and N. Su, Phys. Lett. B 720, 192 (2013).

[27] N. Su and K. Tywoniuk, Phys. Rev. Lett. 114, 161601 (2015).

[28] D. Dudal, J. A. Gracey, S. P. Sorella, N. Vandersickel and H. Verschelde, Phys. Rev. D 78, 065047 (2008).

[29] W. Florkowski, R. Ryblewski, N. Su and K. Tywoniuk, Acta Phys. Polon. B 47, 1833 (2016).

[30] A. Bandyopadhyay, N. Haque, M. G. Mustafa and M. Strickland, Phys. Rev. D 93, 065004 (2016).

[31] W. Florkowski, R. Ryblewski, N. Su and K. Tywoniuk, Phys. Rev. C 94, 044904 (2016).

[32] V. Begun, W. Florkowski and R. Ryblewski, arXiv:1602.08308 [nucl-th]. 